\section{Capital Social: dados descritivos de estudo de base populacional e associação com comportamentos simultâneos de risco à saúde

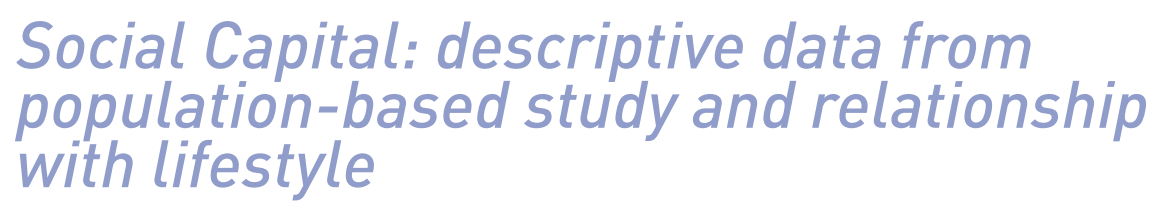

Mathias Roberto Loch ${ }^{1,2}$

Regina Kazue Tanno de Souza'

\section{RESUMO}

O objetivo deste trabalho foi apresentar dados descritivos sobre indicadores de capital social (CS) na população de um município de médio porte do Paraná, Brasil e analisar suas possíveis associações com a exposição simultânea a comportamentos de risco à saúde. Realizou-se estudo transversal de base populacional que incluiu 1.081 pessoas de 40 anos ou mais do município de Cambé-PR. Foram analisados dados sobre indicadores de CS e de quatro comportamentos (inatividade física no lazer, irregular consumo de frutas e verduras, tabagismo e consumo abusivo de álcool), sendo posteriormente verificados a associação entre os indicadores de CS e a quantidade de comportamentos de risco à saúde (dados agregados). Observou-se maior prevalência de nenhum comportamento de risco entre os que haviam participado nos últimos 12 meses de alguma atividade cívica/comunitária ( $15,5 \%$ vs $7,8 \%$ dos que não haviam participado), enquanto a prevalência de três ou quatro comportamentos de risco foi maior entre os que referiram não ter nenhum amigo $(18,1 \%$ vs $24,3 \%$ dos que referiram quatro ou mais amigos) e entre os que percebiam menor frequência de ajuda entre as pessoas do bairro $(23,6 \%$ entre os que consideravam que as pessoas no bairro se ajudam somente algumas vezes, raramente ou nunca $v s 15,5 \%$ daquelas que consideravam que a ajuda entre as pessoas do bairro acontecia sempre ou quase sempre). Em geral, as pessoas com melhores indicadores de CS apresentavam menor quantidade de comportamentos de risco à saúde. Recomenda-se que as políticas públicas de promoção de comportamentos saudáveis se apropriem da ideia/conceito de CS.

\section{PALAVRAS-CHAVE}

Capital social; Estilo de vida; Epidemiologia social; Estudos transversais; Adultos.

\begin{abstract}
The aim of this study was presenting descriptive data on indicators of social capital (SC) in representative sample of the population aged 40 years or more from a mid-size city in southern Brazil and the association of SC indicators with simultaneous exposure to risk bealth behaviors. We conducted a population-based cross-sectional study that included the population of 40 years or more $(n=1081)$. The survey instrument consisted information on indicators of SC and four bealth behaviors (physical inactivity in leisure time, irregular consumption of fruits and vegetables, smoking and alcohol abuse). We verified the association between CS indicators and the amount of bebavior bealth risk (aggregate). Higher prevalence of zero risk behavior was observed among those who participated in the last 12 months some civic or community activity $(15.5 \% \quad 7.8 \%$ of those who had not participated). The prevalence of three or four risk behaviors was higher among those who reported having no friends (18.1\% 24.3\% of those reporting four or more friends) and among those who reported less often belp among people of neighborbood (23.6\% among those who felt that people in the neighborhood to belp only sometimes, rarely or never, $15.5 \%$ of those who that felt belp from people in the neighborbood occurred always or almost always). In general, people with better SC indicators had a lower amount of risk health behaviors. It is recommended that public policies consider the SC as an important aspect in the promotion of healthy behaviors.
\end{abstract}

\section{KEYWORDS}

Social Capital; Life Style; Social Epidemiology; Social Participation; Cross-Sectional Studies; Adults.
Rev Bras Ativ Fis Saúde p. 774-784 DOI

http://dx.doi.org/10.12820/rbafs.v.19n6p774

1 Programa de Pós-Graduação em Saúde Coletiva. Universidade Estadual de Londrina, PR.

2 Departamento de Educação Física. Universidade Estadual de Londrina, PR. 


\section{INTRODUÇÃO}

O conceito de capital social (CS) está ligado às características da organização social, a confiança interpessoal, normas de reciprocidade e redes solidárias, que capacitam os participantes a agir coletivamente e mais eficientemente na busca de objetivos e metas comuns (Pattussi, 2006, a partir de Putnam e Coleman) ${ }^{1}$.

Foi principalmente a partir da publicação dos trabalhos de Putnam que os estudos sobre CS passaram a aumentar em número e terem maior repercussão ${ }^{2}$. Em um de seus trabalhos, Putnam investigou a Itália entre os anos 1860 e 1987 e concluiu que um dos fatores que influenciava o maior desenvolvimento da região Norte daquele país era o engajamento cívico da população em comparação ao menor desenvolvimento e participação da população da região Sul italiana ${ }^{3}$.

Evidentemente existem importantes pontos divergentes entre os diversos autores que elaboraram definições ou na abordagem de CS que adotam. O uso plural do CS acaba suscitando dúvidas metodológicas, o que explica inclusive o fato de que boa parte da produção existente sobre o assunto dedicar-se a explorar seus aspectos conceituais ${ }^{4}$. Por outro lado, apesar de o CS ser um conceito relativamente "elástico", em geral, as definições e abordagens encontradas na literatura possibilitam uma aproximação da ideia geral do conceito e de sua aplicação prática em diversos contextos.

Ademais, apesar de o CS ter potencial de influenciar aspectos positivos como a democracia, o desenvolvimento econômico e social, a relação deste com diversos aspectos da vida humana precisa sempre ser avaliada em cada contexto, inclusive em função da possível influência negativa que ele pode exercer sobre estes mesmos aspectos. Isso acontece principalmente quando o CS serve de elemento para exclusão ou preconceito em relação às pessoas que não fazem parte de um determinado grupo ou compartilham dos mesmos valores. Esta situação pode gerar a exclusão dos outsiders. Assim, a cultura do pertencimento a um determinado grupo social pode também excluir e marginalizar as pessoas que não compartilham dos mesmos valores. Assim, quando o CS promove a exclusão dos "diferentes", pode fomentar a rivalidade e o conflito entre os grupos. Nestes casos desenvolve-se uma espécie de confiança particularizada, que pode gerar CS negativo na forma de racismo, etnocentrismo, intolerância religiosa, etc. ${ }^{2,5,6}$.

Além disso, o CS pode servir para o desenvolvimento de máfias, gangues ou outros grupos ou organizações sociais indesejáveis. Outra crítica importante se dá quando o discurso do CS é utilizado como forma de transferir para a sociedade responsabilidades que deveriam ser do Estado ${ }^{2,4}$. Para Matos $(2009)^{2}$ existem algumas estratégias que podem ser utilizadas para se diminuir os possíveis efeitos negativos do CS. A primeira estratégia deve ser "dar voz" aos cidadãos, fortalecendo-os de maneira que possam expressar publicamente seus problemas e necessidades. Deve-se também buscar aumentar a simetria das obrigações entre os membros, já que a igualdade de obrigações tende a reduzir a vulnerabilidade dos membros. Além disso, deve-se buscar valorizar aspectos de uma democracia plural, enfatizando-se normas inclusivas e universais em detrimento às normas restritas que podem gerar preconceitos de diversas naturezas.

$\mathrm{Na}$ América Latina o conceito de CS, pelo menos na área da saúde, ainda parece ser pouco explorado. Em um estudo de revisão que incluiu somente 
investigações realizadas em populações da América Latina e do Caribe, foram identificados somente 11 artigos originais e concluiu-se que a evidência atual permite esboçar, mas não afirmar, que existe relação entre CS e melhores indicadores de saúde nos países latino-americanos?

Especificamente sobre a relação entre CS e estilo de vida, existem evidências que pessoas com maior CS tendem a ser mais ativas fisicamente ${ }^{8-12}$, apresentam características de alimentação mais saudável ${ }^{9,13}$ e menor prevalência de tabagismo ${ }^{9,12,14}$. De todos os estudos encontrados que investigaram a relação entre indicadores de CS e algum comportamento relacionado à saúde, apenas dois $^{9,15}$ foram realizados na América Latina.

Dado este contexto, o objetivo deste trabalho foi apresentar dados descritivos sobre indicadores CS em uma amostra representativa da população de um município de médio porte do Paraná, Brasil e a associação do CS com a exposição simultânea a comportamentos de risco à saúde.

\section{MÉTODOS}

A população de estudo foi constituída pelos moradores de 40 anos ou mais de idade da área urbana do município de Cambé-PR, município localizado na região Norte do Paraná. Em 2010, Cambé tinha 93.733 habitantes (35.607 com idade igual ou maior que 40 anos) ${ }^{16}$.

Com base na prevalência estimada de $50 \%$ dos fatores de risco o tamanho da amostra foi calculado utilizando-se o aplicativo StatCalc do programa Epi Info 3.5.1, considerando margem de erro de 3 pontos percentuais, intervalo de confiança de $95 \%$, resultando em uma amostra de 1.066 pessoas. Prevendo-se $25 \%$ de eventuais perdas e recusas, o cálculo resultou em uma amostra de 1.332 pessoas. Porém, na divisão proporcional entre os setores censitários, a amostra totalizou 1.339 indivíduos a serem entrevistados devido à aproximação do número para o próximo inteiro.

O plano amostral se deu em três etapas: o primeiro, relacionado ao setor censitário, o segundo em relação ao domicílio, e o terceiro em relação ao morador dos domicílios selecionados. Na primeira etapa, todos os 86 setores censitários da região urbana foram incluídos no estudo. O número de pessoas entrevistadas em cada setor foi calculado proporcionalmente à quantidade de homens e mulheres de 40 anos ou mais de cada setor. Em cada domicilio visitado, era feita a identificação das pessoas com 40 anos ou mais e apenas um morador de cada domicilio era entrevistado. Assim, quando, por exemplo, duas ou mais pessoas na residência tinham o perfil necessário para a amostra, era feito o sorteio de um destes moradores. O morador sorteado, caso concordasse em participar do estudo, assinava o Termo de Consentimento Livre e Esclarecido.

As entrevistas face a face foram realizadas por estudantes de graduação ou pós-graduação do Centro de Ciências da Saúde da Universidade Estadual de Londrina. Todos os entrevistadores passaram por treinamento prévio.

Para a caracterização das pessoas, foram levantadas informações sobre sexo, idade, classe econômica e escolaridade. Para a avaliação da classe econômica, foi utilizada a classificação da Associação Brasileira de Empresas de Pesquisa - ABEP (ABEP, 2008) ${ }^{17}$.

As perguntas realizadas sobre os indicadores de CS fazem parte da versão brasileira do Questionário Integrado para medição do CS (QI-MCS). O 
referido instrumento busca fornecer um conjunto de questões do tipo que permite gerar dados quantitativos sobre diferentes dimensões do CS. Para tal, os indicadores selecionados foram retirados de estudos anteriores sobre CS, em que foi demonstrada sua confiabilidade e validade ${ }^{18}$. Entretanto, no contexto da presente pesquisa, a utilização da íntegra do instrumento não foi possível, pois o mesmo conta com um elevado número de perguntas (102 questões). Assim, a aplicação completa deste instrumento, que permitiria uma maior aproximação com o constructo "capital social" foi considerada inviável, uma vez que o CS era somente um dos objetivos do projeto maior (Vigicardio - Doenças cardiovasculares no Estado do Paraná: mortalidade, perfil de risco, terapia medicamentosa e complicações). Assim, cabe enfatizar que se buscou investigar "indicadores" de CS e não propriamente o constructo CS, que é bastante complexo e exigiria um estudo mais específico. Por outro lado, as perguntas selecionadas possibilitaram o levantamento de indicadores já utilizados em outras investigações $\mathrm{s}^{6,8,10,11,15,19,20}$ e foram selecionados indicadores de CS cognitivo (ligado às percepções ou expectativas do comportamento social e do senso de comunidade) e do CS estrutural (relacionado à partícipação cívica e política).

Os indicadores de capital social e as respectivas categorias utilizadas na análise dos dados foram: número de amigos próximos, considerando as pessoas com quem a pessoa se sente à vontade para conversar a respeito de assuntos particulares, ou chamar caso necessite ajuda (categorias: quatro ou mais; um a três; zero), número de pessoas que emprestariam dinheiro em caso de necessidade (pelo menos uma pessoa; nenhuma pessoa), confiança nas pessoas do bairro (positiva $=$ concorda totalmente ou em parte com a afirmação: "podese confiar na maioria das pessoas que moram neste(a) bairro/localidade"; não concorda, nem discorda, discorda em parte ou discorda totalmente da mesma afirmação), percepção da segurança no bairro (consideram o bairro muito ou moderadamente pacífico; consideram o bairro nem pacífico, nem violento, moderadamente violento ou muito violento), percepção sobre a frequência com que as pessoas no bairro se ajudam (sempre ou quase sempre ajudam; algumas vezes, raramente ou nunca ajudam), e a participação em atividades cívicas ou comunitárias (ter participado ou realizado nos últimos 12 meses pelo menos uma destas atividades: Reunião de conselho, reunião aberta ou grupo de discussão; encontrou, falou ou escreveu para algum político; de algum protesto ou demonstração, campanha eleitoral ou informativa, alertou jornal, rádio ou televisão para algum problema local, notificou a polícia para algum problema local; não participou de nenhuma das atividades mencionadas).

Foram avaliados quatro comportamentos de risco à saúde (inatividade física no lazer, consumo irregular de frutas e verduras, tabagismo e consumo abusivo de álcool). Para a avaliação inatividade física foi utilizado o modelo de estágio de mudança de comportamento, em que foram considerados inativas no lazer as pessoas em fase de pré-contemplação (não tinham intenção de começar a praticar atividade física no lazer nos próximos seis meses), contemplação (pretendiam começar a realizar atividade física no lazer nos próximos seis meses) e preparação (pretendiam começar a fazer atividade física nos próximos trinta dias).

Para a avaliação do consumo irregular de frutas e verduras, foram utilizadas duas perguntas do VIGITEL (BRASIL, 2009), especificamente: a) em 
quantos dias da semana o(a) $\mathrm{Sr}(\mathrm{a})$ costuma comer frutas?; b) Em quantos dias de uma semana o(a) Sr(a) costuma comer pelo menos um tipo de verdura ou legume (alface, tomate, couve, chuchu, berinjela, abobrinha), sem contar batata, mandioca ou inhame? Em ambas as perguntas as opções de resposta eram: nunca; quase nunca; 1 a 2 dias; 3 a 4 dias; 5 a 6 dias; todos os dias. Foram considerados como tendo consumo irregular de frutas e/ou verduras aqueles que referiram, em pelo menos uma das questões: nunca; quase nunca; 1 a 2 dias; ou 3 a 4 dias.

Para avaliação do tabagismo e do consumo abusivo de álcool utilizaram-se as mesmas questões utilizadas pelo VIGITEL. O tabagismo foi avaliado pela seguinte pergunta: $\mathrm{O}$ (a) $\mathrm{Sr}(\mathrm{a})$ fuma? Foram considerados fumantes aqueles que referiram fumar atualmente, independente da quantidade. $\mathrm{O}$ consumo abusivo de álcool foi avaliado, considerando-se o consumo dos últimos 30 dias. Foi realizada a seguinte pergunta: "nos últimos 30 dias o(a) Sr(a) consumiu mais que quatro (para mulheres)/cinco (para homens) doses de bebida alcoólica em uma mesma ocasião? A dose foi definida como uma lata de cerveja, uma taça de vinho ou uma dose de cachaça, uísque ou qualquer outra bebida alcoólica destilada.

Todos os formulários foram checados e posteriormente fez-se a dupla digitação e a comparação dos dois bancos de dados no programa Epi Info® versão 3.5.1. As análises foram realizadas no programa Statistical Package for the Social Sciences® (SPSS) versão 19.0. O teste do qui-quadrado foi utilizado para se investigar possíveis diferenças nas proporções dos indicadores de CS conforme sexo e nível econômico. Foram calculadas as prevalências e respectivos Intervalos de Confiança (IC 95\%) do número de comportamentos de risco à saúde (zero, um, dois, três ou quatro) conforme categorias de CS em cada indicador isolado de CS, ajustada pelas seguintes variáveis: sexo, faixa etária, classe econômica, estado nutricional, dificuldade de deslocamento, consulta médica nos últimos 12 meses e autopercepção de saúde.

O Comitê de Ética em Pesquisa da Universidade Estadual de Londrina aprovou todos os procedimentos metodológicos do trabalho (parecer 236/10). Outras informações metodológicas podem ser obtidas em outros trabalhos ${ }^{9,22}$.

\section{RESULTADOS}

Do total de 1.339 pessoas elegíveis, 1.180 (88,1\%) foram entrevistados. Ocorreram 159 perdas $(11,9 \%)$. Para a análise do presente estudo, foram excluídas as pessoas que não responderam a uma ou mais questões relacionadas aos indicadores de CS ou das variáveis demográficas $(n=99)$.

A maior parte da amostra referiu viver com parceiro(a), apresentou baixo nível econômico (61,7\% classe econômica $\mathrm{C}$ ou inferior) e educacional (72,5\% estudaram oito anos ou menos), apresentava excesso de peso $(68,4 \%$ com IMC igual ou superior a $25 \mathrm{~kg} / \mathrm{m}^{2}$ ) e uma autopercepção muito boa ou boa de saúde $(57,8 \%)$ (Tabela 1$)$

Quanto aos indicadores de CS, observou-se que cerca de metade dos entrevistados referiu ter quatro amigos ou mais (49,4\%) e havia participado, nos últimos 12 meses, de alguma atividade cívica/comunitária (48,2\%). A maior parte referiu ter pelo menos uma pessoa que emprestaria dinheiro em caso de necessidade $(72,5 \%)$ e considerava o bairro pacífico (68,5\%). Por outro lado, menos da metade das pessoas investigadas confiava na maioria das pessoas do 
bairro $(42,1 \%)$ e percebiam que as pessoas se ajudavam algumas vezes/quase sempre/sempre (45,5\%). Quando estratificados os indicadores de CS por sexo, observou-se que os homens referiram, em maior proporção do que as mulheres, ter quatro amigos ou mais e percebiam o bairro como pacífico. Nos demais indicadores, não houve diferença entre os sexos (Tabela 2).

TABELA 1 - Características demográficas, Índice de Massa Corporal e autopercepção de saúde, n=1.081, Cambé, Paraná, 2011.

\begin{tabular}{|c|c|c|c|c|c|c|}
\hline \multirow{2}{*}{ Variável } & \multicolumn{2}{|c|}{ Todos } & \multicolumn{2}{|c|}{ Homens } & \multicolumn{2}{|c|}{ Mulheres } \\
\hline & $n$ & $\%$ & $n$ & $\%$ & $n$ & $\%$ \\
\hline \multicolumn{7}{|l|}{ Situação conjugal } \\
\hline Com parceiro & 803 & 74,3 & 426 & 85,7 & 377 & 64,6 \\
\hline Sem parceiro & 278 & 25,7 & 71 & 14,3 & 207 & 35,4 \\
\hline \multicolumn{7}{|l|}{ Classe econômica } \\
\hline A-B (mais alta) & 415 & 38,4 & 215 & 43,3 & 200 & 34,2 \\
\hline C & 567 & 52,5 & 257 & 51,7 & 310 & 53,1 \\
\hline D-E (mais baixa) & 99 & 9,2 & 25 & 5,0 & 74 & 12,7 \\
\hline \multicolumn{7}{|l|}{ Nível educacional } \\
\hline 9 anos ou mais & 297 & 27,5 & 132 & 26,6 & 165 & 28,3 \\
\hline 5 a 8 anos & 272 & 25,2 & 143 & 28,8 & 129 & 22,1 \\
\hline Menos de 5 anos & 511 & 47,3 & 222 & 44,7 & 289 & 49,6 \\
\hline \multicolumn{7}{|l|}{ Índice de masa corporal } \\
\hline Normal $\left[<25 \mathrm{~kg} / \mathrm{m}^{2}\right]$ & 342 & 31,6 & 182 & 36,6 & 160 & 27,4 \\
\hline Sobrepeso $\left(25-29,9 \mathrm{~kg} / \mathrm{m}^{2}\right)$ & 413 & 38,2 & 206 & 41,4 & 207 & 35,4 \\
\hline Obesidade $\left(\geqslant 30 \mathrm{~kg} / \mathrm{m}^{2}\right)$ & 326 & 30,2 & 109 & 21,9 & 217 & 37,2 \\
\hline \multicolumn{7}{|l|}{ Autopercepção de saúde } \\
\hline Muito boa/Boa & 625 & 57,8 & 324 & 65,2 & 301 & 51,5 \\
\hline Regular/Ruim/Muito ruim & 456 & 42,2 & 173 & 34,8 & 283 & 48,5 \\
\hline
\end{tabular}

TABELA 2 - Indicadores de Capital Social. Total e estratificado por sexo, n=1.081, Cambé, Paraná, 2011.

\begin{tabular}{|c|c|c|c|c|c|c|c|}
\hline & \multicolumn{2}{|c|}{ Total } & \multicolumn{2}{|c|}{ Homens } & \multicolumn{2}{|c|}{ Mulheres } & \multirow[t]{2}{*}{$p^{*}$} \\
\hline & $\mathrm{n}$ & $\%$ & $n$ & $\%$ & $n$ & $\%$ & \\
\hline Tem quatro amigos ou + amigo & 534 & 49,4 & 290 & 58,4 & 244 & 41,8 & 0,001 \\
\hline $\begin{array}{l}\text { Tem alguém que emprestaria dinheiro } \\
\text { em caso de necessidade }\end{array}$ & 784 & 72,5 & 370 & 74,4 & 414 & 70,9 & 0,192 \\
\hline Confia na maioria das pessoas do bairro & 455 & 42,1 & 222 & 44,7 & 233 & 39,9 & 0,113 \\
\hline $\begin{array}{l}\text { Percebe que as pessoas no bairro se } \\
\text { ajudam sempre ou quase sempre }\end{array}$ & 492 & 45,5 & 234 & 47,1 & 258 & 44,2 & 0,339 \\
\hline Considera o bairro pacífico & 740 & 68,5 & 361 & 72,6 & 379 & 64,9 & 0,006 \\
\hline $\begin{array}{l}\text { Participou nos últimos } 12 \text { meses de pelo } \\
\text { menos uma atividade cívica/comunitária }\end{array}$ & 521 & 48,2 & 239 & 48,1 & 282 & 48,3 & 0,948 \\
\hline
\end{tabular}

*valor de p considerando as diferenças nas proporções entre os sexos.

Também foi verificada a relação entre a classe econômica e os indicadores de CS. Observou-se associação entre todos os indicadores de CS, exceto frequência com que as pessoas no bairro se ajudam. Em todos os casos, o grupo da maior classe econômica referiu melhores indicadores de CS (Tabela 3). 
TABELA 3 - Indicadores de capital social. Total e estratificado por classe econômica , n=1.081, Cambé, Paraná, 2011.

\begin{tabular}{|c|c|c|c|c|c|c|c|}
\hline & \multicolumn{2}{|c|}{$A B$} & \multicolumn{2}{|c|}{ C } & \multicolumn{2}{|c|}{$\mathrm{DE}$} & \multirow[b]{2}{*}{ p* } \\
\hline & $\mathrm{n}$ & $\%$ & $\mathrm{n}$ & $\%$ & $\mathrm{n}$ & $\%$ & \\
\hline Tem quatro amigos ou + & 229 & 55,2 & 259 & 45,7 & 46 & 46,4 & 0,009 \\
\hline $\begin{array}{l}\text { Tem alguém que emprestaria dinheiro } \\
\text { em caso de necessidade }\end{array}$ & 327 & 78,8 & 397 & 70,0 & 60 & 60,6 & 0,000 \\
\hline Confia na maioria das pessoas do bairro & 200 & 48,2 & 217 & 38,3 & 38 & 38,4 & 0,004 \\
\hline $\begin{array}{l}\text { Percebe que as pessoas no bairro se } \\
\text { ajudam sempre ou quase sempre }\end{array}$ & 202 & 48,7 & 246 & 43,4 & 44 & 44,4 & 0,166 \\
\hline Considera o bairro pacífico & 321 & 77,3 & 358 & 63,1 & 61 & 61,6 & 0,000 \\
\hline $\begin{array}{l}\text { Participou nos últimos } 12 \text { meses de pelo } \\
\text { menos uma atividade cívica/comunitária }\end{array}$ & 258 & 62,2 & 233 & 41,4 & 30 & 30,3 & 0,000 \\
\hline
\end{tabular}

* valor de p de tendência linear considerando as diferenças nas proporções entre as classes econômicas.

Considerando a prevalência simultânea de comportamentos de risco à saúde foi observado que a proporção de pessoas que não apresentava nenhum dos quatro comportamentos de risco foi maior entre os que tinham participado de alguma atividade cívica e/ou comunitária nos últimos 12 meses. No outro extremo, ou seja, pessoas que apresentavam três ou quatro comportamentos de risco, foi constatado maior chance entre os que não tinham nenhum amigo e entre aqueles que percebiam que as pessoas no bairro somente algumas vezes, raramente ou nunca ajudavam umas as outras. Vale mencionar ainda que, numericamente, as maiores prevalências de nenhum comportamento de risco à saúde, foram observadas entre as pessoas com melhores indicadores de CS (em todos os indicadores de CS). Por outro lado, a prevalência de três ou quatro comportamentos de risco à saúde foi numericamente, também em todos os indicadores de CS, maior entre aqueles com piores indicadores de CS.(Tabela 4).

TABELA 4 - Exposição simultânea a comportamentos de risco à saúde, segundo indicadores de capital social, n=1.081, Cambé, Paraná, 2011.

\begin{tabular}{|c|c|c|c|c|}
\hline \multirow{3}{*}{ Variáveis } & \multicolumn{4}{|c|}{ Exposição simultânea a comportamento de risco } \\
\hline & Zero & 1 & 2 & 3 ou 4 \\
\hline & $\%$ (IC95\%) & $\%$ (IC95\%) & $\%$ (IC95\%) & $\%$ (IC95\%) \\
\hline Total & 11,5 & 29,4 & 39,2 & 19,9 \\
\hline \multicolumn{5}{|l|}{ Número de amigos } \\
\hline Quatro ou mais & $13,3(10,6-16,0)$ & $31,1(27,3-34,8)$ & $37,5(33,4-41,7)$ & $18,1(14,9-21,3)$ \\
\hline Um a três & $8,7(5,7-11,8)$ & $30,4(26,2-34,7)$ & $40,0(35,3-44,8)$ & $20,8(17,2-24,4)$ \\
\hline Nenhum & $12,9(7,4-18,4)$ & $19,2(11,5-26,9)$ & $43,6(35,1-52,1)$ & $24,3(17,8-30,8)$ \\
\hline \multicolumn{5}{|l|}{ Dinheiro emprestado } \\
\hline Uma ou mais pessoas & $12,0(9,8-14,2)$ & $30,4(27,4-33,5)$ & $38,9(35,5-42,3)$ & $18,7(16,1-21,3)$ \\
\hline Nenhuma pessoa & $10,1(6,5-13,7)$ & $26,7(21,7-31,7)$ & $40,1(34,5-45,6)$ & $23,1(18,8-27,4)$ \\
\hline \multicolumn{5}{|c|}{ Confiança nas pessoas do bairro } \\
\hline Positivo & $12,4(9,5-15,3)$ & $32,9(28,8-36,9)$ & $36,5(32,0-40,9)$ & $18,3(14,8-21,8)$ \\
\hline Negativo & $10,8(8,3-13,3)$ & $26,9(23,4-30,4)$ & $41,2(37,4-45,1)$ & $21,0(18,1-24,0)$ \\
\hline \multicolumn{5}{|l|}{ Frequência de ajuda } \\
\hline Sempre/Quase sempre & $12,7(9,9-15,5)$ & $34,7(30,8-38,5)$ & $37,2(32,9-41,5)$ & $15,5(12,2-18,8)$ \\
\hline $\begin{array}{l}\text { Algumas vezes/ } \\
\text { raramente/nunca }\end{array}$ & $10,8(8,3-13,3)$ & $25,0(21,5-28,6)$ & $40,9(37,0-44,9)$ & $23,6(20,6-26,6)$ \\
\hline \multicolumn{5}{|l|}{ Segurança no bairro } \\
\hline Pacífico & $12,4(10,1-14,6)$ & $31,5(28,3-34,7)$ & $37,3(33,8-40,8)$ & $18,8(16,1-21,5)$ \\
\hline Violento/Médio & $9,5(6,2-12,9)$ & $24,9(20,1-29,6)$ & $43,5(38,3-48,7)$ & $22,2(18,1-26,2)$ \\
\hline \multicolumn{5}{|l|}{ Participação comunitária } \\
\hline Sim & $15,5(12,7-18,2)$ & $31,0(27,1-34,8)$ & $35,6(31,3-39,8)$ & $18,0(14,8-21,3)$ \\
\hline Não & $7,8(5,1-10,4)$ & $28,0(24,3-31,7)$ & $42,6(38,6-46,7)$ & $21,6(18,5-24,8)$ \\
\hline
\end{tabular}

IC: Intervalo de confiança; Prevalência ajustada por sexo, faixa etária, classe econômica, estado nutricional, dificuldade de deslocamento, consulta médica nos últimos 12 meses e auto-percepção de saúde. 


\section{DISCUSSÃO}

Este trabalho buscou apresentar dados descritivos sobre indicadores de CS e avaliar a associação destes com a exposição simultânea a comportamentos de risco à saúde em um estudo de base populacional, realizado em uma cidade da região Sul do Brasil. Apesar de ser muito difícil determinar de maneira precisa o que seria um "baixo" ou "alto" nível de CS, foi possível observar que, de maneira geral, as pessoas com melhores indicadores de CS apresentavam menor quantidade de comportamentos de risco à saúde. Além disso, quanto maior era a classe econômica, melhores eram os indicadores de CS e que, quando estratificado por sexo, observou-se que os homens referiram ter maior número de amigos e percebiam que o bairro era mais pacífico do que as mulheres.

Apesar de haver sido utilizado um critério bastante amplo de participação comunitária/cívica, pouco menos da metade das pessoas havia participado de alguma atividade desta natureza (entre as atividades investigadas) nos últimos 12 meses. Evidentemente que não se pode desconsiderar que nos últimos anos tem se fortalecido outras formas de CS, e que as mesmas não foram investigadas no presente trabalho. A própria ideia de comunidade em alguma medida é afetada pelas novas possibilidades de comunicação, expandindo os limites geográficos. Em muitos casos, as pessoas podem estar em contato com outras através das redes virtuais, que podem também ter um papel importante enquanto redes de apoio $^{18}$. Entretanto, dado o perfil da população investigada, é pouco provável que muitas pessoas tenham exercido estas novas possibilidades de participação.

A participação comunitária/cívica pode envolver uma grande gama de atividades: atuação em vários tipos de associações comunitárias, clubes de esporte, lazer e arte, partidos políticos, sindicatos, cooperativas, entre outros. Estas participações tendem a aumentar as possibilidades de cooperação horizontal entre os membros, já que o grau de pertencimento pode promover regras de reciprocidade, redimensionando a confiança e possibilitando colaborações entre os participantes ${ }^{4}$. Em geral, nas sociedades com forte presença do Estado nas áreas econômicas e sociais, caso, por exemplo, dos países escandinavos, há maior confiança dos cidadãos para com o Estado. Esta mesma situação não é observada em muitos países onde não existe esta mesma participação do Estado, e o mesmo acaba sendo visto, por vezes, com certa desconfiança pelos cidadãos ${ }^{4}$. No caso brasileiro, Zaluar (2007) ${ }^{23}$ observa várias manifestações de CS negativo, mas reconhece que nas últimas décadas ocorreu um importante aumento no número de organizações da sociedade civil voltadas para fins públicos.

Em função de suas características e história, o Sistema Único de Saúde (SUS) pode exercer um papel importante no fortalecimento da democracia brasileira $^{24}$, inclusive para que o princípio do controle social, entendido como a participação da comunidade no processo decisório e de fiscalização, seja efetivado. Segundo Bahia (2007) ${ }^{25}$ o chamado Movimento da Reforma Sanitária uniu diferentes movimentos sociais ao redor de alguns objetivos comuns, que acabaram resultando nas diretrizes e princípios do SUS. Assim, não se pode ignorar que o próprio SUS é também fruto de uma organização coletiva importante e pode também ser relevante no sentido de desenvolver o CS entre a população brasileira.

Neste trabalho, observou-se que quase todos os indicadores de CS investigados diminuíam à medida que se diminuía a classe econômica. Este dado re- 
força a ideia de que são necessárias políticas públicas que, baseadas no princípio da equidade, enfatizem ações e estratégias para os grupos mais vulneráveis, no sentido inclusive de garantir a participação destes no processo decisório e de fiscalização. Neste sentido, o fortalecimento do CS pode ser importante. Segundo o relatório da Comissão Nacional sobre Determinantes Sociais da Saúde ${ }^{26}$, o desgaste do CS é um mecanismo importante para que as iniquidades sociais e econômicas impactem de forma negativa na situação de saúde das comunidades.

Evidentemente a tarefa de "construir" CS não é simples. Para Putnam³, é possível modificar hábitos e valores de forma a produzir CS, porém lembra que estas modificações tendem a acontecer em um prazo longo. Para Portes $(2000)^{27}$, as redes sociais não são dadas naturalmente, e precisam ser construídas por meio de estratégias orientadas para a institucionalização das relações do grupo.

Quanto à associação entre o nível de CS e a prevalência de três ou quatro comportamentos de risco à saúde, observou-se que as pessoas com maior CS tinham menor prevalência desta característica. Com relação aos mecanismos pelos quais se dá a relação entre CS e o estilo de vida, vale mencionar que estes não estão plenamente esclarecidos. Kawachi e Berkman (2000) ${ }^{28}$ apontam três hipóteses: o CS promoveria uma mais rápida difusão da informação, aumentaria a probabilidade de que os comportamentos saudáveis fossem adotados como normas e exerce algum tipo de controle social sobre os comportamentos considerados de risco à saúde.

Assim, considerando que as políticas públicas de promoção da saúde no Brasil tem buscado abordar também a questão das mudanças comportamentais, considera-se pertinente recomendar que estas políticas considerem a questão do CS, e busquem inclusive o fortalecimento das redes e da participação comunitária, que podem ter potencial de contribuir com o "afastamento" do discurso do estilo de vida das criticadas abordagens excessivamente normativas e descontextualizadas. Desse modo, as políticas de promoção de comportamentos saudáveis devem se pautar em estratégias que busquem não a coerção e o excesso de normatização (que pode ter efeitos mais prejudiciais do que benéficos), mas sim um convencimento sobre a importância dos hábitos saudáveis e a criação efetiva de condições objetivas para que a adoção destes comportamentos seja mais fácil por parte da população, ao mesmo tempo que preservem as liberdades e escolhas individuais.

Algumas limitações e delimitações do estudo precisam ser destacadas, entre as quais a dificuldade operacional para se medir o constructo CS e o fato de incluirmos apenas pessoas com 40 anos ou mais. Por outro lado, este estudo buscou apresentar um tema ainda pouco investigado e debatido no contexto das políticas públicas de promoção da saúde no Brasil. Evidentemente a temática do CS nas políticas/intervenções relacionadas à saúde ainda é incipiente e muitos aspectos ainda precisam ser melhor investigados/esclarecidos. Entender melhor como se dá a influência entre o CS e o estilo de vida, e mesmo a influência do CS na efetividade de programas relacionados à saúde, pode ser importante. Também compreender em que medida alguns programas, por exemplo, os programas formais de atividade física oferecidos em Unidades Básicas de Saúde, podem auxiliar na criação de maiores níveis de CS entre os membros da comunidade, pode ser um foco interessante de estudos.

Dado este contexto e os resultados do presente estudo, considera-se pertinente recomendar que as políticas que buscam a promoção de comporta- 
mentos saudáveis se apropriem da ideia/conceito de CS. É claro que estas políticas não podem partir do princípio que o CS seja por si só e a priori sempre positivo. Não devem ignorar os possíveis efeitos negativos do CS, que podem ocorrer principalmente quando são aplicadas normas restritas ao invés de valorizadas normas inclusivas e universais. O discurso do CS também não deve servir para substituir o papel do Estado. É preciso lembrar que, segundo a Constituição Brasileira, a saúde é considerada um direito de todos e dever do Estado. Evidentemente, este "dever do Estado" pode ser mais bem realizado com a participação das comunidades e sociedades. Outro ponto é não atribuir exclusivamente à baixa organização da comunidade e/ou participação dos seus membros a responsabilidade por eventuais problemas. Há de se considerar as características sociais de cada comunidade em uma perspectiva histórica e com uma compreensão não simplista dos fenômenos sociais existentes.

\section{Agradecimento}

À Coordenação de Aperfeiçoamento de Pessoal de Nível Superior (CAPES) pela concessão da bolsa do Programa Institucional de Doutorado Sanduíche no Exterior (processo n ${ }^{\circ}$ 7242-12-3).

\section{REFERÊNCIAS}

1. Pattussi MP, Moyses SJ, Junges JR, Sheiham A. Capital Social e a agenda da epidemiologia. Cad Saude Publica. 2006;22(8):1525-46.

2. Matos H. Capital Social e comunicação: interfaces e articulações. São Paulo: Summus Editorial; 2009.

3. Putnam R. Comunidade e democracia: a experiência da Itália moderna. Rio de Janeiro: Editora FGV; 1998.

4. D'Araújo MD. Capital Social. Rio de Janeiro: Jorge Zahar; 2003.

5. Uphoff EP, Pickett KE, Cabieses B, Small N, Wright J. A systematic review of the relationships between social capital and socioeconomic inequalities in health: a contribution to understanding the psychosocial pathway of health inequalities. Int $\mathrm{J}$ Equity Health. 2013;12:54.

6. Hurtado D, Kawachi I, Sudarsky J. Social capital and self-rated health in Colombia: the good, the bad and the ugly. Soc Sci Med. 2011;72(4):584-90.

7. Kripper CE, Sapag JC. [Social capital and health in Latin America and the Caribbean: a systematic review]. Rev Panam Salud Publica. 2009;25(2):162-70.

8. Lindstrom M, Hanson BS, Ostergren PO. Socioeconomic differences in leisure-time physical activity: the role of social participation and social capital in shaping health related behaviour. Soc Sci Med. 2001;52(3):441-51.

9. Loch MR. Associação entre capital social e comportamentos relacionados à saúde: estudo de base populacional. Londrina: Doutorado em Saúde Coleitva. Universidade Estadual de Londrina; 2013.

10. Mummery WK, Lauder W, Schofield G, Caperchione C. Associations between physical inactivity and a measure of social capital in a sample of Queensland adults. J Sci Med Sport. 2008;11(3):308-15.

11. Ueshima K, Fujiwara T, Takao S, Suzuki E, Iwase T, Doi H, et al. Does social capital promote physical activity? A population-based study in Japan. PLoS One. 2010;5(8):e12135.

12. Ball K, Jeffery RW, Abbott G, McNaughton SA, Crawford D. Is healthy behavior contagious: associations of social norms with physical activity and healthy eating. Int J Behav Nutr Phys Act. 2010;7:86.

13. Johnson CM, Sharkey JR, Dean WR. Eating Behaviors and Social Capital are Associated with Fruit and Vegetable Intake Among Rural Adults. J Hunger Environ Nutr. 2010;5(3):302-15. 
14. Lindstrom M, Moghaddassi M, Bolin K, Lindgren B, Merlo J. Social participation, social capital and daily tobacco smoking: a population-based multilevel analysis in Malmo, Sweden. Scand J Public Health. 2003;31(6):444-50.

15. Sapag JC, Poblete FC, Eicher C, Aracena M, Caneo C, Vera G, et al. Tobacco smoking in urban neighborhoods: exploring social capital as a protective factor in Santiago, Chile. Nicotine Tob Res. 2010;12(9):927-36.

16. Brasil. Instituto Brasileiro de Geografia e Estatística. Censo Demográfico 2010. 2010.

17. Associação Brasileira das Empresas de Pesquisas - Critério de classificação econômica. 2008.

18. Grootaert C, Narayan D, Nylan V, Wookcook JM. Acessado em 2003

19. Lindstrom M. Social capital, desire to increase physical activity and leisure-time physical activity: a population-based study. Public Health. 2011;125(7):442-7.

20. Mohnen SM, Volker B, Flap H, Groenewegen PP. Health-related behavior as a mechanism behind the relationship between neighborhood social capital and individual health--a multilevel analysis. BMC Public Health. 2012;12:116.

21. Brasil. Vigitel Brasil 2010: vigilância de fatores de risco e proteção para doenças crônicas por inquérito telefônico. Brasília: Ministério da Saúde, 2011.

22. Souza RKT, Bortoletto MSS, Loch MR, González AD, Matsuo T, Cabrera MAS, et al. Prevalência de fatores de risco cardiovascular em pessoas com 40 anos ou mais de idade, em Cambé, Paraná (2011): estudo de base populacional. Epidemiol Serv Saude. 2013;22(3):435-44.

23. Zaluar A. A democratização inacabada: fracasso da segurança pública. Estudos Avançados. 2007;21(61):31-49.

24. Bastos FA, Santos E, Ferreira M. Capital Social e Sistema Único de Saúde (SUS) no Brasil. Saúde e Sociedade. 2009;18(2):177-88.

25. Bahia L, Costa NR, Van Stralen C. A saúde na agênda pública: convergências e lacunas nas pautas de trabalho das instituições governamentais e movimentos sociais. Cien Saude Colet. 2007;12(Supl.):1791-818.

26. Brasil. Comissão Nacional sobre determinantes sociais da Saúde - Relatório Final da Comissão Nacional sobre determinantes sociais da Saúde. 2008.

27. Portes A. Capital Social: origens e aplicações na sociologia contemporânea. Sociologia, Problemas e práticas. 2000;33(1):133-58.

28. Kawachi I, Berkman L. Social Cohesion, Social Capital and Health. In: Berkman L, Kawachi, I., editor. Social Epidemiology. Oxford: Oxford University Press; 2000.

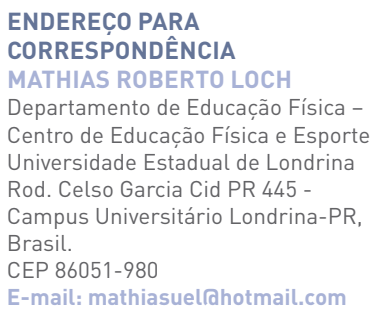

